\title{
Molecular Dynamics and Hyperpolarization Performance of Deuterated $\beta$-Cyclodextrins
}

\author{
Filippo Caracciolo, ${ }^{\dagger}$ Efstathios Charlaftis, ${ }^{\dagger}$ Lucio Melone, $^{\ddagger}$ and Pietro Carretta ${ }^{*} \dagger$ \\ $\dagger$ Department of Physics, University of Pavia, 27100-Pavia, Italy \\ $\ddagger$ Department of Chemistry, Materials, and Chemical Engineering G. Natta, Politecnico di \\ Milano, 20133-Milano, Italy \\ E-mail: pietro.carretta@unipv.it
}

\section{Supplementary Information}

$\beta$-CD (Alfa Aesar) was dried in oven at $105{ }^{\circ} \mathrm{C}$ for $12 \mathrm{~h}$ before use. Odomethane- ${ }^{13} \mathrm{C}, \mathrm{D}_{3}$ was purchased from Sigma-Aldrich. Dimethylformamide (DMF) was dried over $\mathrm{CaH}_{2}$ before use. Other solvents and reagents used in this work are commercially available and were used as received. MeCD-TEMPO was synthesized as reported in a previous work. ${ }^{1} \mathrm{H}$-NMR (Fig. 1) and ${ }^{13} \mathrm{C}-\mathrm{NMR}$ (Fig. 2) characterization were carried out at $305 \mathrm{~K}$ with a Bruker Avance-500 MHz NMR spectrometer (Bruker, Billerica, MA, USA). ESI-MS mass spectra were collected on a Bruker Esquire $3000+$ with electrospray ionization source and ion-trap detector. The samples were analyzed by direct infusion of suitable solutions (methanol) in the spectrometer source. 


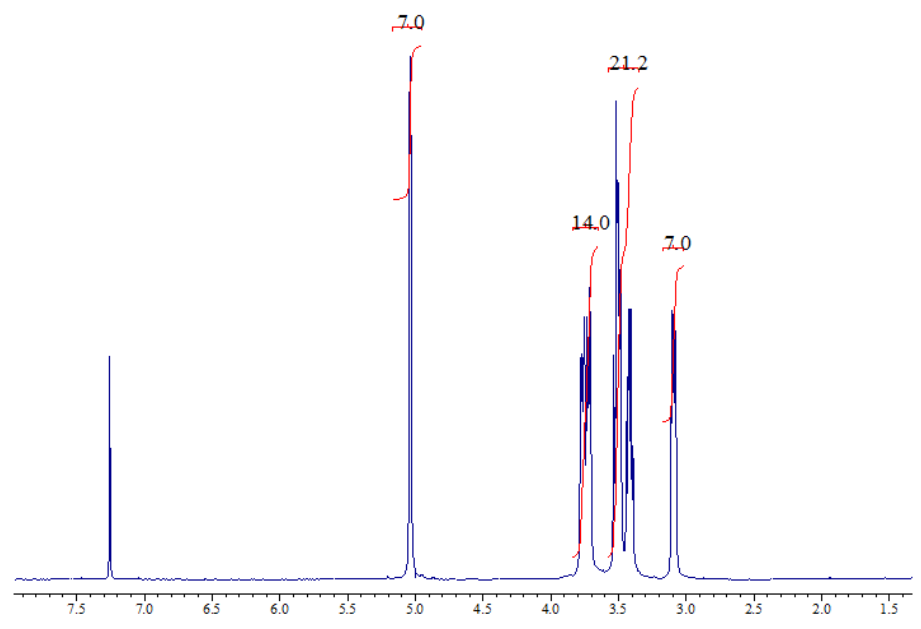

Figure S1: ${ }^{1} \mathrm{H}-\mathrm{NMR}$ spectrum of CD21d (solvent: $\mathrm{CDCl}_{3}$ ).

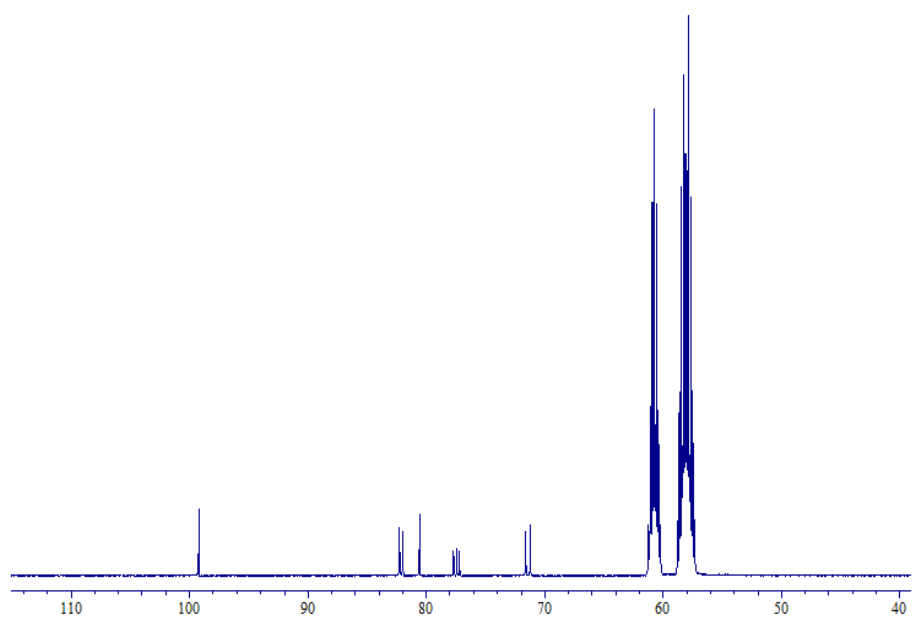

Figure S2: ${ }^{13} \mathrm{C}-\mathrm{NMR}$ spectrum of CD21d (solvent: $\mathrm{CDCl}_{3}$ ).

For the synthesis of the sample CD21d, $227 \mathrm{mg}$ of $\beta$-CD $(0.20 \mathrm{mmol})$ were dissolved in $5 \mathrm{~mL}$ of DMF. $600 \mathrm{mg}$ of sodium hydride (60\% in mineral oil) were added. The mixture was stirred at $0{ }^{\circ} \mathrm{C}$ for $30 \mathrm{~min}$ under $\mathrm{N}_{2}$. Finally, $1.0 \mathrm{~g}$ of ${ }^{13} \mathrm{CD}_{3} \mathrm{I}(6.85 \mathrm{mmol})$ was added. After $24 \mathrm{~h}$ at $25^{\circ} \mathrm{C}$ and under $\mathrm{N}_{2}$, the excess $\mathrm{NaH}$ was eliminated with water and the product was extracted with chloroform. Purification on $\mathrm{SiO}_{2}$ column $\left(\mathrm{CHCl}_{3}: \mathrm{MeOH}, 10: 0.5\right)$ afforded the pure product as a white foam after solvent evaporation (220 mg, yield:72\%). ESI-MS: $[\mathrm{M}+\mathrm{Na}]+$, calculated 1536.15 , found 1536.50 , see Fig. 3 . 


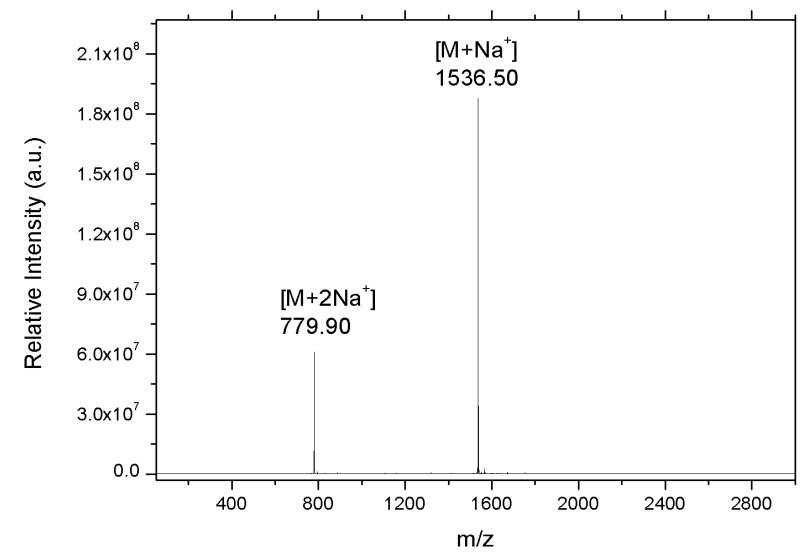

Figure S3: ESI-MS spectrum of the sample CD21d in positive mode.

For the preparation of CD21Rd, $83.2 \mathrm{mg}$ of CD21d and $10.2 \mathrm{mg}$ of MeCD-TEMPO were dissolved in $400 \mu \mathrm{L}$ of methanol and stirred for $10 \mathrm{~min}$. Solid CD21Rd (1.03\% of TEMPO radical, see eq. (1) was obtained after solvent evaporation under vacuum (see Fig. 4).

$$
\% \text { TEMPO }=100 \times \frac{\frac{M e C D-T E M P O}{1650.82} \times 156.25}{C D 21+(M e C D-T E M P O)}
$$

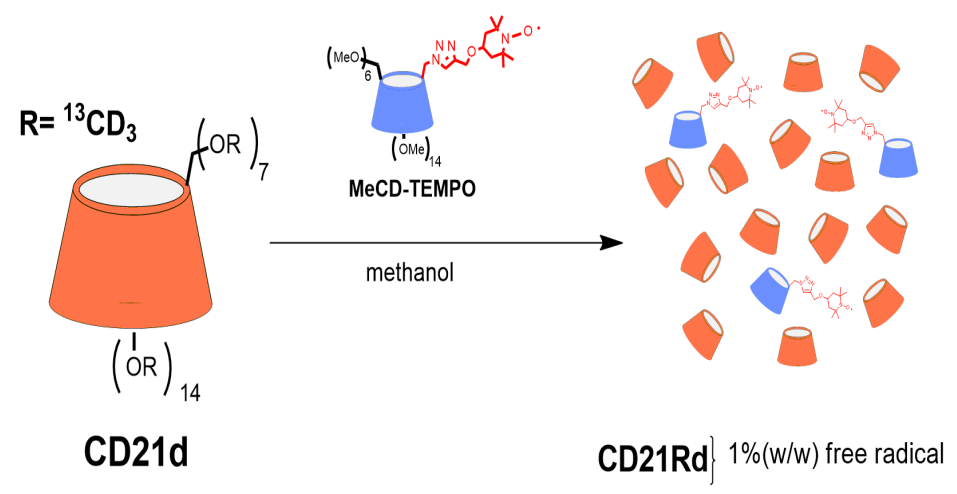

Figure S4: Preparation of the sample CD21Rd. 


\section{References}

(1) F. Caracciolo, A. Lucini Paioni, M. Filibian, L. Melone, P. Carretta, Proton and Carbon-13 Dynamic Nuclear Polarization of Methylated $\beta$-Cyclodextrins, J.

Phys. Chem. B, 2018, 122, 1836-1845 